\title{
Controlling the Volume of the Focal Cerebral Ischemic Lesion through Photothrombosis
}

\author{
Tiannan Wang ${ }^{1,2}$, Wenju Cui ${ }^{1,2}$, Yicheng Xie ${ }^{1,2}$, Weiping Zhang ${ }^{2,3}$, Shinghua Ding ${ }^{1,2^{*}}$ \\ ${ }^{1}$ Dept. of Biological Engineering, ${ }^{2}$ Dalton Cardiovascular Research Center, University of Missouri, Columbia, \\ MO 65211 \\ ${ }^{3}$ Dept. of Pharmacology, School of Medicine, Zhejiang University, Hangzhou, 310058, China. \\ *Corresponding author \\ Shinghua Ding \\ Dalton Cardiovascular Research Center \\ Dept. of Biological Engineering \\ University of Missouri-Columbia \\ 134 Research Park Drive \\ Columbia, MO 65211. \\ Phone: (573) 884-2489. \\ Fax: (573) 884-4232. \\ E-mail: dings@missouri.edu.
}

\begin{tabular}{l|l|l} 
Received: 31 July 2009; & Revised: 16 August 2009; & Accepted: 24 August 2009
\end{tabular}

\begin{abstract}
Stroke is the third leading cause of human death. Various models have been used to study the mechanisms of tissue damage and neuronal protection. Each model has its advantages and disadvantages. Middle cerebral artery occlusion (MCAo), has been widely used, but produces large ischemic lesions of varying size, which is a disadvantage in the study of neuronal protective mechanisms. Photothrombosis provides an alternative model that can produce consistent lesion size. In this model, ischemia is induced by photo-activation of rose bengal in blood flow by green light illumination. Because ischemia can be induced while animals are kept in a stereotaxic device or on the stage of a microscope, this model has become widely used for in vivo study of cellular structure and function. In this study, photothrombosis was generated by using a wide-field epi-fluorescent microscope equipped with a metal halide lamp and ischemia was confirmed by blood flow reduction monitored by laser Doppler flowmetry. We demonstrated, by adjusting the intensity of output light and the size of irradiated area in the cortex, that the photothrombosis model can, with high reproducibility, be used to generate ischemic infarction of various sizes in mouse brains. Using immunostaining and histochemistry of brain sections, our data showed that photothrombosis induced neuronal death and that brain infarct volume was correlated with the power output and the size of irradiated area of the cortex.
\end{abstract}

Keywords: infarction; rose bengal; power output; laser Doppler; penumbra. 


\section{Introduction}

Stroke is the third-leading cause of human death in the United States. Cerebral ischemia accounts for approximately $80 \%$ of all human strokes and has a major impact on public health[1]. It causes primary neuronal death in the ischemic core, and leads to delayed neuronal death and neuronal degeneration in the penumbra[2]. Several animal models have been established for the study of pathogenesis after ischemia; middle cerebral artery occlusion (MCAo) is the one that has been the most widely used[1;3;4]. However, this model has been criticized in that it circumvents the participation of platelet aggregation which is a primary initiator of ischemic events. The production of ischemia in this model depends on an invasive and sophisticated surgical approach. Another disadvantage of MCAo is the variability of infarct size due to different degrees of collateral blood flow. It also destroys much of the forebrain cortex. The photothrombosis model[5-7], based on the photochemically stimulated platelet aggregation by rose bengal, overcomes these drawbacks. In addition, this model can produce infarction in specific regions of interest in the cortex. Inflammatory response and apoptosis following photothrombosis have been characterized[8;9]. Recently this model has been used for in vivo two-photon imaging to monitor structural and functional plasticity of neurons as well as $\mathrm{Ca}^{2+}$ signaling in astrocytes using timelapse studies before and after photothrombosis[7;10-12], which is very difficult using the MCAo model. In clinical settings, the size of lesion and severity of brain damage varies among ischemic patients, thus an ischemia model that can control the sizes and severity of the damaged region would provide a good tool to study the pathology and neuronal protection of ischemia. Here we studied the effect of light power and the size of irradiated area on brain damage following photothrombosis. Using fluorescence microscopy, the size of irradiated area and the intensity of light can be readily controlled by adjusting the field aperture along the optical pathway and the output power of the metal halide lamp used for inducing photothrombosis.
Neuronal death and cellular response were assessed and characterized using histochemistry and immunocytochemistry. Our results demonstrated that the infarct volumes were correlated with the power of light and the size of irradiated area.

\section{Materials and Methods}

\subsection{Animals}

Male FVB/NJ mice 6-8 weeks of age were purchased from The Jackson Laboratory (Bar Harbor, MA). All procedures were performed in accordance with the NIH Guide for the Care and Use of Laboratory Animals and were approved by the University of Missouri Animal Care Quality Assurance Committee.

\subsection{Craniotomy surgery and photothrombosis}

Mice were anesthetized with an intraperitoneal (i.p.) injection of ketamine/xylazine $(130 \mathrm{mg} / 10 \mathrm{mg} / \mathrm{kg}$ body weight) dissolved in artificial cerebral spinal fluid (ACSF) (in $\mathrm{mM}$ ): $120 \mathrm{NaCl}, 10$ Hepes, $5 \mathrm{KCl}, 3.1 \mathrm{CaCl}_{2}$, $1.3 \mathrm{MgCl}_{2}, 10$ glucose and 10 Hepes, $\mathrm{pH}$ 7.4. Once the animal reached a surgical level of anesthesia, it was placed on a warm heating pad to maintain body temperature at $37^{\circ} \mathrm{C}$ for surgery. A small incision was made on the scalp, and a circular craniotomy $(2.0 \mathrm{~mm}$ in diameter $)$ was made using a high speed drill over the somatosensory cortex at the coordinate of $-0.8 \mathrm{~mm}$ from bregma and $2.0 \mathrm{~mm}$ lateral to the midline. The photosensitive dye rose bengal (RB) dissolved in ACSF was then injected into mice through the tail vein at a dose of $0.03 \mathrm{mg} / \mathrm{g}$ body weight. To induce photothrombosis, an area in the middle of the craniotomy was focally irradiated with a green light $(560 \pm 20 \mathrm{~nm})$ from a X-cite $120 \mathrm{PC}$ metal halide lamp (EXFO, Canada) for 2 min through a $10 \times 0.3 \mathrm{NA}$ objective (Nikon, Japan). We chose this wavelength range because rose bengal has a peak absorption wavelength of $560 \mathrm{~nm}[13]$. The skin was sutured after induction of photothrombosis and mice were sent back to the animal facility after recovery. The intensity of light can be adjusted by selecting the output level of the lamp, and the size of irradiated area in the cortex can be controlled by adjusting the () 2010 by NWPII. All rights reserved. 
diaphragm of the field aperture of the microscope along the optical pathway which has been calibrated by a reticle in the eyepiece.

\subsection{Power measurement of lamp}

Power output from the metal halide lamp was measured using a Model 407A power meter (Spectra-Physics, CA). The light was filtered using a filter cube with bandwidth $560 \pm 20 \mathrm{~nm}$ and was focused on the power meter using a 10x objective. The data were the average from four different measurements.

\subsection{Regional cerebral blood flow (CBF) measurement with laser Doppler flowmetry (LDF)}

After mice were anesthetized with a mixture of ketamine and xylazine, a fiber-optic probe connected to a laser-Doppler flowmeter (Model MBF3D, Moor Instruments, UK) was placed at the center of cranial window over the barrel field in somatosensory cortex using a micromanipulator (Narishige, Japan). The blood flow was continuously monitored starting from $10 \mathrm{~min}$ before the induction of photothrombosis to $60 \mathrm{~min}$ afterwards. The manipulator can accurately control the 3D position. The probe was positioned close to cortical surface in cranial window. After established the baseline $\mathrm{CBF}$, the probe was moved to the side and the cranial window was irradiated. The probe was moved back to the same position immediately after irradiation. The $\mathrm{CBF}$ was continuously monitored for another 60 minutes after irradiation. The decrease in percentage of $\mathrm{CBF}$ was calculated using the baseline $\mathrm{CBF}$ before photothrombosis. During the surgery of cranial window we applied warm $\operatorname{ACSF}\left(37^{\circ} \mathrm{C}\right)$ on the surface to avoid the decrease of the cortical temperature. In addition, there is a lamp also close to the animal to keep the animal warm. After photothrombosis, animals were kept on a heating pad to maintain body temperature at $37^{\circ} \mathrm{C}$ until recovery.

\subsection{Transcardial perfusion and immunostaining}

The procedure for transcardial perfusion has been described previously[14]. Briefly, mice were anesthetized with halothane and transcardially perfused first with ice-cold phosphate buffered saline (PBS) and then 4\% paraformaldehyde in PBS ( $\mathrm{pH}=7.4)$. After perfusion, the brain was post-fixed with $4 \%$ paraformaldehyde in PBS at $4^{\circ} \mathrm{C}$ for $30 \mathrm{~min}$, and then transferred to $30 \%$ sucrose overnight to prevent ice crystal formation. Coronal sections of the brain (thickness $20 \mu \mathrm{m}$ ) were cut on a cryostat (Leica CM 1900, Germany), and were collected serially on precleaned glass slides.

NeuN and fluoro-Jade B (FJB) stainings for neurons have been described previously[12;14]. Briefly, floating brain sections were incubated with rabbit anti-NeuN (1:1000, Millipore, CA) overnight, and subsequently incubated with fluorescein isothiocyanate (FITC)-conjugated goat anti-rabbit IgG (Millipore, CA) for $2 \mathrm{hr}$. Similar procedures were used for glial fibrillary acidic protein (GFAP) and Ibal staining using a rabbit polyclonal anti-GFAP (1:150, Sigma, MO) and a rabbit anti-Iba1 polyclonal antibody (1:600, Wako Pure Chemical Industries). Nuclei were stained with 4'-6-Diamidino-2-phenylindole (DAPI) (0.5 $\mu \mathrm{g} / \mathrm{ml}$ in PBS) and the brain sections were finally mounted on glass slides using anti-fade mounting medium. For detecting degenerating neurons, brain sections on the glass slide were stained with $0.0004 \%$ FJB for $30 \mathrm{~min}$. Although floating section staining give much better results it is difficult to avoid the loss of tissue in the core region due to the damage of the tissue. Since in our experiment we control the lesion in small size with the maximal of $2 \mathrm{~mm}$ diameter for irradiation, the edema in brain section was not obvious.

Images of the stained sections were acquired using a Nikon epi-fluorescence microscopy system controlled by Metamorph Imaging software (Molecular Device, CA). Excitation was generated by an X-cite 120 PC metal halide lamp (EXFO, Canada). Emission was detected by a CoolSNAP-EZ CCD-camera (Photometrics, AZ).

\subsection{Nissl staining and infarct volume measurement}

For Nissl staining, the $20 \mu \mathrm{m}$ coronal brain sections were immersed in $0.2 \%$ cresyl violet/acetic acid solution for $2 \mathrm{~min}$, followed by two 5-minute washes with $95 \%$ ethanol. They were then dehydrated in $100 \%$ ethanol followed (C) 2010 by NWPII. All rights reserved. 
by cleaning in xylene and mounted on a glass slide with mounting medium. The microphotographs of Nissl staining were taken with an Olympus microscope. The infarct volume was determined by measuring areas showing the loss of Nisslstaining in brain sections[8;15]. The areas of cerebral infarction were delineated and quantified using the ImageJ software (NIH). The total volume of ischemic tissue was calculated by multiplying the individual infarct area in the consecutive sections by the thickness of sections.

\section{Results}

\subsection{Induction of Ischemia by Photothrombosis}

Photothrombosis is induced by illumination of the cortex after the photo-sensitive chemical rose bengal is injected into the circulatory system. Therefore, we first calibrated the light powers at focal plane of a 10x objective in our microscope. The levels of power output (as percentage of maximal output) of the lamp and aperture sizes were measured and the results are shown in Table 1.

Previously, we used two-photon microscopy to measure blood flow changes in capillaries and whisker-stimulated field potential before and after photothrombosis[12]. Since two-photon imaging is only suitable for blood flow measurement of capillaries, not for large blood vessels, and blood flow rates might be different in small and large blood vessels, these measurements do not represent the overall blood flow change of the ischemic region. In this study we used a laser Doppler flowmetry to measure the CBF before and after photothrombosis to confirm the induction of ischemia (Fig. 1A). In this experiment, we used $50 \%$ power level with $1 \mathrm{~mm}$ diameter of illuminating area as an example for blood flow measurement. The CBF was continuously monitored starting from $10 \mathrm{~min}$ before photothrombosis to establish the baseline and up to $60 \mathrm{~min}$ thereafter. The $\mathrm{CBF}$ was reduced to $40 \%$ of baseline immediately after photothrombosis and recovered slightly to about $48 \%$ of baseline after $30 \mathrm{~min}$, presumably due to reperfusion. Since the probe senses blood flow in a 3D volume which may not be homogeneous after photothrombosis it is conceivable that the resultant $\mathrm{CBF}$ only represent the overall reduction in the $3 \mathrm{D}$ region and thus it may not necessarily reduce to zero. The data demonstrate that photothrombosis successfully induced ischemia and are consistent with our previous study using two-photon imaging to monitor blood flow of capillaries[12].

Table 1. Light power measurements with different settings of light output from a metal halide lamp and different sizes of field apertures

\begin{tabular}{cccc}
\hline Light output $^{1}$ & Power $(\mathbf{m W})$ & Aperture size $^{2}$ & Power $\left.^{\mathbf{m W}}\right)$ \\
\hline $12.5 \%(1 \mathrm{~mm})$ & $0.124 \pm 0.006$ & $0.5 \mathrm{~mm}(25 \%)$ & $0.060 \pm 0.001$ \\
$25 \%(1 \mathrm{~mm})$ & $0.226 \pm 0.006$ & $1.0 \mathrm{~mm}(25 \%)$ & $0.226 \pm 0.006$ \\
$50 \%(1 \mathrm{~mm})$ & $0.482 \pm 0.020$ & $2.0 \mathrm{~mm}(25 \%)$ & $0.884 \pm 0.011$
\end{tabular}

1-In this set of experiment, the sizes of irradiated area were fixed at $1 \mathrm{~mm}$ in diameter. 2-In this set of experiment, light outputs were fixed to $25 \%$. The data were average from four different measurements and expressed as mean \pm se.

Using Nissl staining, we examined the formation of ischemic lesions under ischemic conditions of $25 \%$ light output and $1 \mathrm{~mm}$ diameter of irradiation area. The animals were sacrificed at $24 \mathrm{hr}$ after photothrombosis. Fig. 1B shows representative images of a series of rostro-caudal brain coronal sections with ischemic lesions. The lesion was well demarcated and a consistent pattern of brain damage was observed, suggesting that, like other ischemic models, photothrombosis can reliably induce an ischemic lesion.

\subsection{The effect of power levels on infarct volume}


Next we studied the effect of power of irradiating light on brain damage. Photothrombosis of the mouse cortex was induced by irradiating the cortex for 2 min with various power outputs from the metal halide lamp in different groups of mice. The sizes of irradiated regions were kept constant at $1 \mathrm{~mm}$ in diameter. One day after photothrombosis, the mice were anesthetized and transcardially perfused, and the brains were sectioned and stained with cresyl violet to assess brain damage. Figs. 2A-C show the Nissl-stained brain sections in the middle of ischemic lesions under different conditions. It is somewhat surprising that with the power output of $12.5 \%$ for the induction of photothrombosis, no clear demarcated infarction was observed, thus we were unable to measure infarct volume for this condition (Fig. 2A). However, well-demarcated infarction surrounded by vital tissue was observed with power outputs of $25 \%$ and $50 \%$ (Fig. 2B \& C). Further analysis shows that the infarct volume increases with increasing power output (Fig. 2D). These data demonstrate that brain damage induced by photothrombosis can be controlled by light power. No lesion was observed in the contralateral side of the cortex of mice subject to photothrombosis or the cortex irradiated with light alone without rose bengal injection (data not shown), demonstrating that rose bengal or light irradiation per se does not cause brain damage and the combination of rose bengal injection and light irradiation is required to produce ischemic damage.
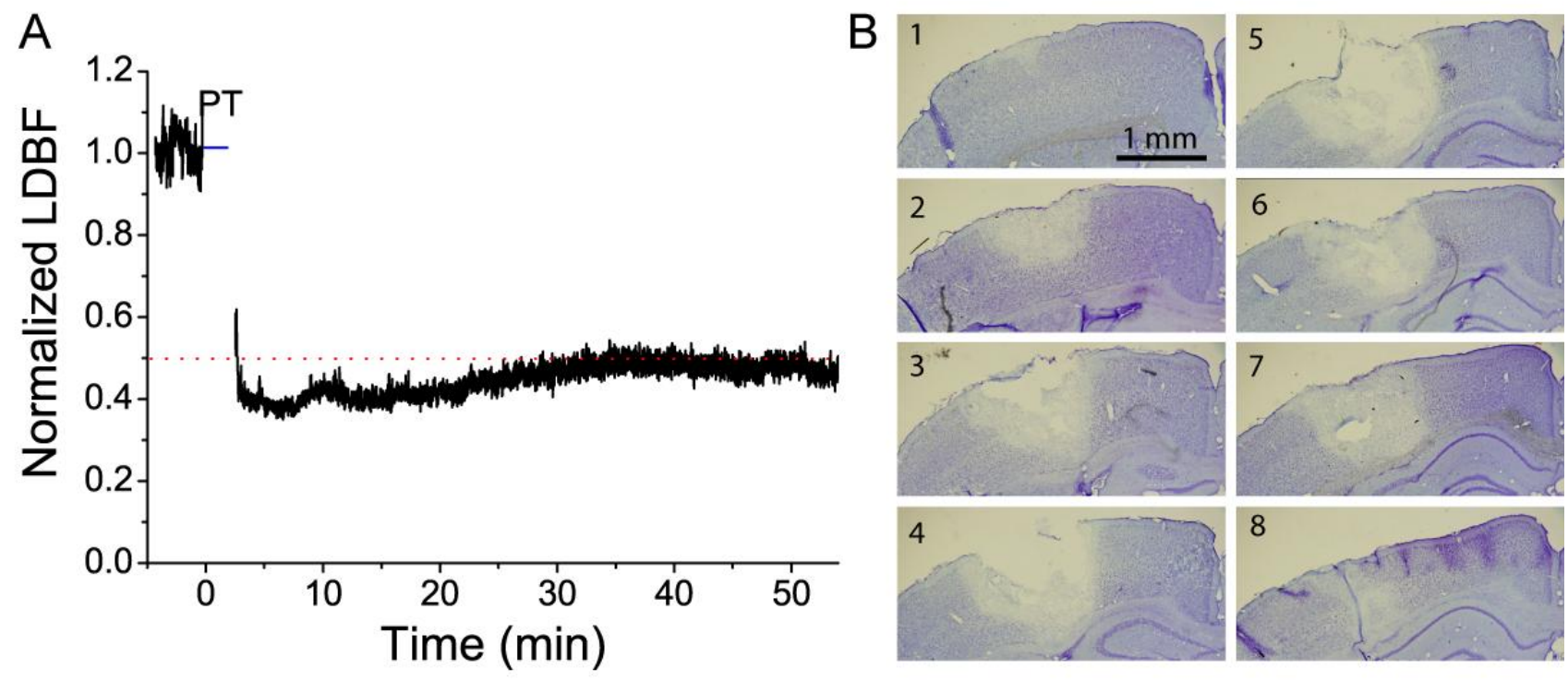

Figure 1: Photothrombosis induced ischemia and brain damage. A) Cerebral blood flow (CBF) measurement with a laser Doppler flowmetry. CBF was reduced to $40 \%$ of baseline immediately following photothrombosis. In this experiment, photothrombosis was induced by 2 min irradiation of $50 \%$ power output on a $1 \mathrm{~mm}$ area in cranial window. CBF was monitored up to $1 \mathrm{hr}$ after photothrombosis and a similar value was maintained. Data were averaged from $\mathrm{N}=5$ mice. B) Representative rostro-caudal series of Nissl-stained coronal sections from a mouse after photothrombosis. Photothrombosis was induced in a region of $1 \mathrm{~mm}$ of diameter with $25 \%$ of lamp power output. The mouse was sacrificed at $24 \mathrm{hr}$ after photothrombosis. The interval between each section is $0.2 \mathrm{~mm}$.

\subsection{The effect of the size of irradiated area on ischemic lesion}

Because of observed variation in infarction size in stroke patients, we next examined the effect of the size of the irradiated area on the brain lesion. Since lamp power of $25 \%$ produced a clear demarcated lesion, we chose this power to induce ischemia with varying sizes of irradiating area. After rose bengal was injected, mouse cranial windows were irradiated for 2 min. Areas $(0.5,1$, and $2 \mathrm{~mm}$ in diameter) of exposure were varied by adjusting the field aperture of the microscope. 
Each area of exposure was made in a separate group of mice. The size of irradiated area was calibrated with a reticle in the eyepiece of the microscope. Mice were sacrificed $24 \mathrm{hr}$ later and brain sections were stained with cresyl violet to assess brain damage as before. In each case, a distinct lesion with a sharp transition zone was observed (Fig. 3A-C). The infarct volume increased with the area of irradiated cortical region (Fig. 3D).
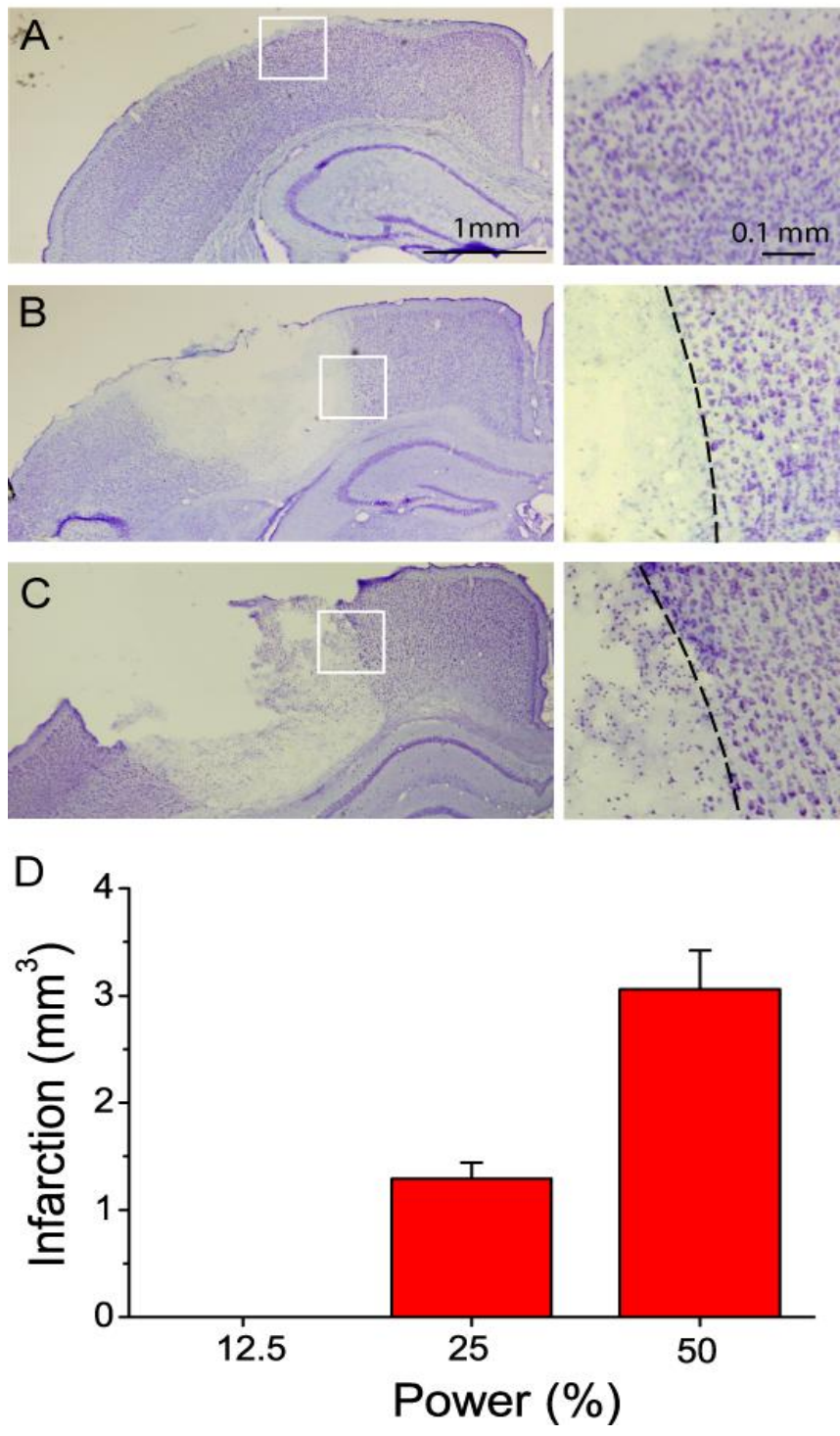

Figure 2: The effect of power of irradiating light on photothrombosis-induced brain damage. A-C) (Left) Nissl staining revealed infarct and transition zones in the cortex caused by photothrombosis with $12.5 \%$, $25 \%$ and $50 \%$ power outputs. The images were taken from brain sections in the middle of infarct region.
(Right) High-resolution images from the boxed regions in the left panels show clear transition zones and demarcation. Note: there is no detectable lesion in the brain sections from mice that were illuminated with $12.5 \%$ power. D) The effect of power output for the induction of photothrombosis on infarct volume. Data were averaged from $\mathrm{N}=4-6$ mice for each condition.
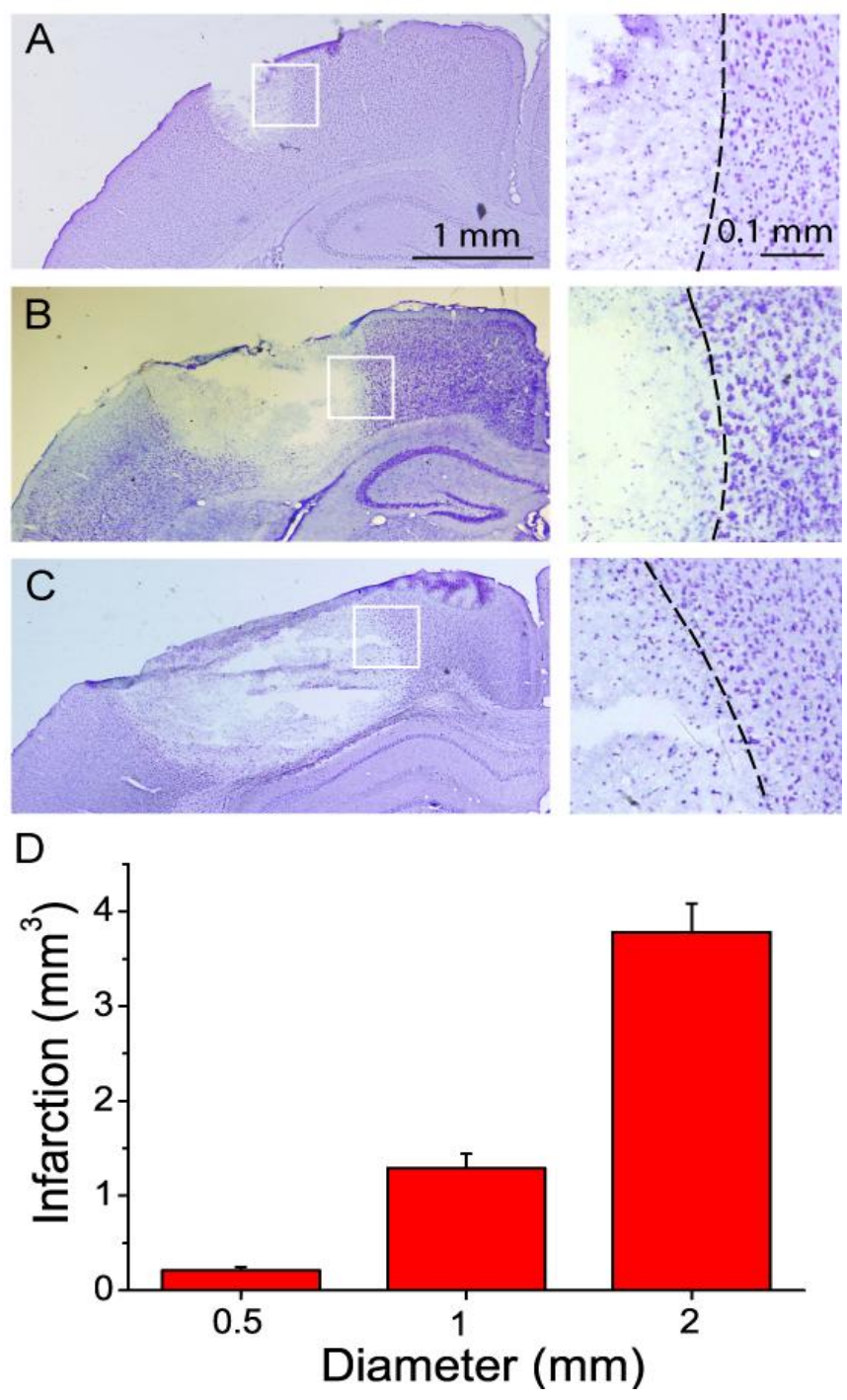

Figure 3: The effect of the size of irradiated area on photothrombosis-induced brain damage. A-C) Brain sections with Nissl staining revealed infarction in the cortex with different sizes caused by photothrombosis with $0.5 \mathrm{~mm}, 1 \mathrm{~mm}$ and $2 \mathrm{~mm}$ of irradiation diameter. The images were taken from brain sections in the middle of infarct region. High resolution images from the boxed regions in the left panels show clear transition zones and demarcation. D) The effect of irradiated diameter in cranial window on infarct volumes. Data were averaged from $\mathrm{N}=4-6$ mice for each condition. 

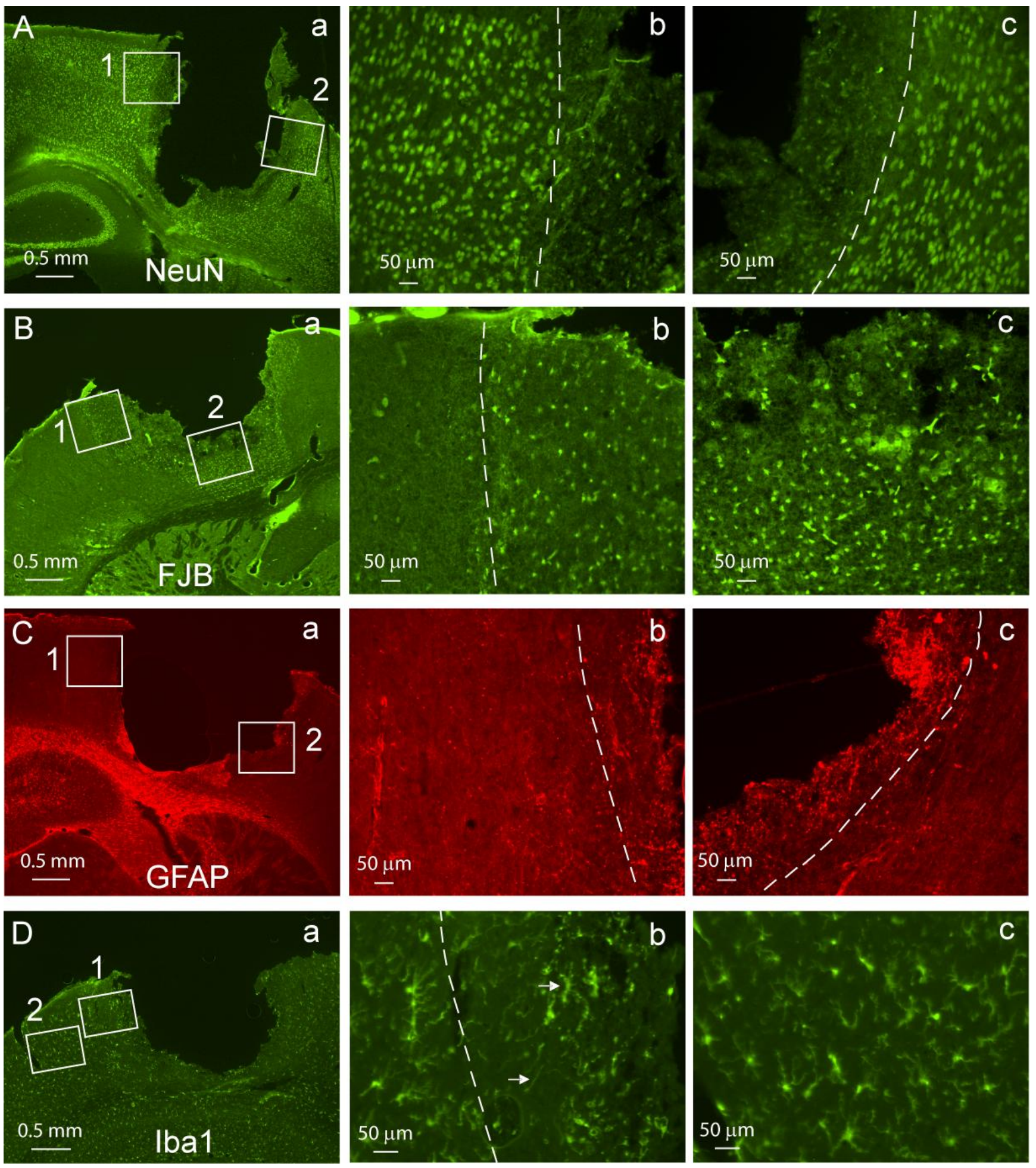

Figure 4: Photothrombosis caused neuronal death and glial cell activation. A) NeuN staining of a brain section showing neuronal loss in the ischemic region and healthy neurons in penumbra. (a) A low resolution image. (b-c) High resolution images of the boxed regions in (a). B) FJB staining for degenerating neurons in core and penumbra. (a) A low resolution image. (b-c) High resolution images of the boxed regions in (a). C) Enhanced GFAP expression in the transition zone between ischemic core and penumbra (right) after photothrombosis. (a) A low resolution image. (b-c) High resolution images of the boxed regions in (a). C) Microglia activation in the penumbra. (a) A low resolution image. (b-c) High resolution images in the penumbra (b) and normal region (c). The arrows indicate the activated microglia with different morphologies. Dash lines in A-D outline the infarction border.

Am. J. Biomed. Sci. 2010, 2(1), 33-42; doi: 10.5099/aj100100033 @ 2010 by NWPII. All rights reserved. 


\subsection{Photothrombosis caused neuronal death and glial cell activation}

To further study the cellular response after photothrombosis, we performed immunostaining on brain sections with ischemic damage induced by the power output of $25 \%$, with an irradiated area of $1 \mathrm{~mm}$ in diameter. Brain sections were immunostained with an antibody against NeuN, a neuronal-specific marker. As shown in Fig. 4A, neuronal losses were clearly seen in the infarct region at $24 \mathrm{hr}$ after photothrombosis as evidenced by the reduction or absence of NeuN immunoreactivity. A sharp transition from infarction to normal tissue was observed (delineated by dash lines in Fig. 4A (b-c)). FJB staining, which is commonly used for detecting degenerating neurons, shows many FJB+ cells in the infarction (Fig. 4B), with a sharp transition to the normal region (Fig. $4 \mathrm{~B}(\mathrm{~b}-\mathrm{c})$ ). We also observed the increased immunoreactivity of GFAP in the penumbra (Fig. 4C), indicating the activation of astrocytes after photothrombosis. However, an individual GFAP+ cell was rarely identified, suggesting astrocyte reactivation did not occur completely at this post-ischemia time point. This can be confirmed by the observation that GFAP expression level is significantly increased and individual GFAP+ cells can be identified in the penumbra a few days after photothrombosis (data not shown). In addition, astrocytes in the cortex do not express high level of GFAP under normal condition (see undamaged region in Fig. 3C). Iba1 is a specific microglia marker, which can stain both resting and activated microglia. The resting microglia showed relatively small cell body with multiple processes. The microglia near the penumbra either has an enlarged cell body with shorter and fewer processes or has elongated processes (indicated with arrows Fig. 4D (b)), suggesting the activation of microglia after photothrombosis. The morphology of microglia distant from ischemic region is the same as that of microglia located in the contralateral side of ischemia. These phenomena were observed in all mice subjected to immunostaining examination. Although the middle parts of infarction in brain section were lost due to the approach of floating-section staining, the transition from normal tissue to infarction was clearly observed in each type of staining.

\section{Discussion}

An animal model of focal cerebral ischemia, providing both reproducibility and precise control of lesion size, is critical for translational studies. The MCAo model produces a large infarction volume which may not model smaller and more survivable strokes, and the variation in lesion size of this model tends to be large, ranging from 5\% to $50 \%$ of cerebral hemisphere[16], which is disadvantageous for neuroprotective studies. Photothrombosis-induced ischemia provides a model that can meet the requirements of reproducibility and small lesion size. However, although this model has been used for more than two decades, how irradiating light power and size of irradiated area affect infarction have not been systematically studied. Here we showed that photothrombosis can generate different sizes of lesions by adjusting the light output and the size of irradiated area using epi-fluorescence microscopy. Our data demonstrated that severity and infarction are correlated with the intensity of irradiating light and the size of irradiated area. It is interesting to note that no observable lesion was produced using lamp power of $12.5 \%$ for inducing photothrombosis. This result suggests two possibilities. First, there might be a threshold power for inducing significant brain-tissue damage. Second, this level of irradiation causes only minor injury that is repaired within 24 hours. The ischemic lesion is also proportional to the diameter of irradiating area, providing a flexible approach to generate the different sizes of ischemic lesions.

In the clinical setting, stroke patients are likely to have lesions of varying sizes and thus may require different therapeutic approaches and care. Therefore, developing a model that can control the ischemic lesion is important for mechanistic studies of neuronal death and neuroprotective studies. The photothrombosis model is less technically demanding and is possible for most laboratories (with fluorescence microscopes) to generate, thus providing a good model for mechanistic studies of neuronal 
protection using varying lesion size. In addition, due to the small lesion size, the animals are able to maintain normal physiological parameters [7;12].

In order to be less invasive, several studies have induced photothrombosis through intact skull[8;9;17-19]. In this study, we induced photothrombosis within the cranial window because it is accessible for in vivo imaging using two-photon microscopy. Light scatters and is reflected when the intact skull is illuminated; for this reason, the infarction produced by photothrombosis using a cranial window instead might likely be more sharply demarcated and have a more consistent lesion size than using intact skull. Because of the convenience of ischemic induction, this model recently has been used to monitor molecular and cellular events before and after ischemic insult in vivo using two-photon microscopy[7;11;12;14;20]. Varying severity of ischemic injury will have varying effects on neuronal and glial function. Thus by changing the light intensity, a variety of pathophysiological phenomena initiated by ischemia can be studied using two-photon microscopy. Using this model, it is now possible to monitor phenomena such as extracellular glutamate accumulation, neuronal and glial $\mathrm{Ca}^{2+}$ signaling, and cellular structural changes in vivo[7;12]. While this model has its advantages, it still has limitations. For example, it is not feasible to control reperfusion, thus tissue damage might come from either ischemic and reperfusion injury, or purely ischemic injury. Small capillaries might be permanently occluded, but large vessels will possibly get reperfused depending on the strength of photothrombosis. In addition, this model has a small penumbral region. Although for histological studies, photothrombosis can be induced through intact skull, for in vivo imaging a craniotomy is required. Surgery itself does not cause lesions, however.

In summary, our study demonstrated that the volume of the focal ischemic lesion can be controlled by manipulating the intensity of irradiating light and the size of irradiated area in the cranial window. Importantly, the approach relied on the use of a wide-field epi-fluorescent microscope equipped with a metal halide lamp, available in most laboratories, providing a flexible and convenient method for developing an animal model for focal ischemia. This model can be used for neuroprotective studies as well as in vivo imaging to perform time-lapse studies on pathophysiological phenomena associated with acute or chronic ischemia.

\section{Acknowledgements}

The work was supported by the grant from American Heart Association (0735133N) and startup funds to SD. We thank Cynthia Haydon for critical reading of the manuscript.

\section{References}

(1) Stapf C, Mohr JP. Ischemic stroke therapy. Annual Review of Medicine 2002; 53:453475.

(2) Barber PA, Demchuk AMH. Biochemistry Ischemic Stroke. Advances in Neurology 2003; 92:151-164.

(3) Karpiak ST, Wakade CG, Karpiak SE, Tagliavia A, Wakade CG. Animal models for the study of drugs in ischemic stroke. Annu 1989; Rev. Pharmacol. Toxicol. 1989; 29:403-429.

(4) Stoll G, Jander S, Schroeter M. Inflammation and glial responses in ischemic brain lesions. Progress in Neurobiology 1998; 56:149-171.

(5) Watson BD, Dietrich WD. Animal models in stroke. Stroke 1990; 21:1376-1377.

(6) Watson BD, Dietrich WD, Busto R, Wachtel MS, Ginsberg MD. Induction of reproducible brain infarction by photochemically initiated thrombosis. Annals of Neurology 1985; 17:497-504.

(7) Zhang S, Boyd J, Delaney K, Murphy TH. Rapid Reversible Changes in Dendritic Spine Structure In Vivo Gated by the Degree of Ischemia. J Neurosci 2005; 25:5333-5338.

(8) Schroeter M, Jander S, Stoll G. Non-invasive induction of focal cerebral ischemia in mice by photothrombosis of cortical microvessels: characterization of inflammatory responses. Journal of Neuroscience Methods 2002; 117:43-49.

(9) Eichenbaum JW, Pevsner PH, Pivawer G, Kleinman GM, Chiriboga L, Stern A, Rosenbach A, Iannuzzi K, Miller DC. A 
murine photochemical stroke model with histologic correlates of apoptotic and nonapoptotic mechanisms. Journal of Pharmacological and Toxicological Methods 2002; 47:67-71.

(10) Brown CE, Li P, Boyd JD, Delaney KR, Murphy TH. Extensive Turnover of Dendritic Spines and Vascular Remodeling in Cortical Tissues Recovering from Stroke. J Neurosci 2007; 27:4101-4109.

(11) Murphy TH, Li P, Betts K, Liu R. TwoPhoton Imaging of Stroke Onset In Vivo Reveals That NMDA-Receptor Independent Ischemic Depolarization Is the Major Cause of Rapid Reversible Damage to Dendrites and Spines. J Neurosci 2008; 28:1756-1772.

(12) Ding S, Wang T, Cui W, Haydon PG. Photothrombosis ischemia stimulates a sustained astrocytic $\mathrm{Ca}^{2+}$ signaling in vivo. GLIA 2009; 57:676-776.

(13) Hai Du R-CAFJL. A Computer-Aided Design and Research Tool in Photochemistry Photochemistry and Photobiology. 1998; 68:141-2.

(14) Ding S, Fellin T, Zhu Y, Lee SY, Auberson YP, Meaney DF, Coulter DA, Carmignoto G, Haydon PG. Enhanced Astrocytic $\mathrm{Ca}^{2+}$ Signals Contribute to Neuronal Excitotoxicity after Status Epilepticus. J Neurosci 2007; 27:10674-10684.

(15) Vendrame M, Cassady J, Newcomb J, Butler T, Pennypacker KR, Zigova T, Davis Sanberg
C, Sanberg PR, Willing AE. Infusion of Human Umbilical Cord Blood Cells in a Rat Model of Stroke Dose-Dependently Rescues Behavioral Deficits and Reduces Infarct Volume. Stroke 2004; 35:2390-2395.

(16) Carmichael ST, Carmichael ST. Rodent models of focal stroke: size, mechanism, and purpose. NeuroRx 2005; 2:396-409.

(17) Lee JK, Park MS, Kim YS, Moon KS, Joo SP, Kim TS, Kim JH, Kim SH. Photochemically induced cerebral ischemia in a mouse model. Surgical Neurology 2007; 67:620-625.

(18) Pevsner PH, Eichenbaum JW, Miller DC, Pivawer G, Eichenbaum KD, Stern A, Zakian KL, Koutcher JA. A photothrombotic model of small early ischemic infarcts in the rat brain with histologic and MRI correlation. Journal of Pharmacological and Toxicological Methods 2001; 45:227-233.

(19) Dietrich WD, Ginsberg MD, Busto R, Watson BD. Photochemically induced cortical infarction in the rat. 1. Time course of hemodynamic consequences. Journal of Cerebral Blood Flow \& Metabolism 1986; 6:184-194.

(20) Winship IR, Murphy TH. In Vivo Calcium Imaging Reveals Functional Rewiring of Single Somatosensory Neurons after Stroke. J Neurosci 2008; 28:6592-6606. 